\title{
Predicting outcomes in older ED patients with influenza in real time using a big data- driven and machine learning approach to the hospital information system
}

Tian-Hoe Tan ${ }^{1}$, Chien-Chin Hsu ${ }^{1,2}$, Chia-Jung Chen ${ }^{3}$, Shu-Lien Hsu', Tzu-Lan Liư ${ }^{3}$, Hung-Jung Lin ${ }^{1,5}$, Jhi-Joung Wang ${ }^{6,7}$, Chung-Feng Liu ${ }^{6+}$ and Chien-Cheng Huang ${ }^{1,8, *^{*}+}$ (D)

\begin{abstract}
Background: Predicting outcomes in older patients with influenza in the emergency department (ED) by machine learning (ML) has never been implemented. Therefore, we conducted this study to clarify the clinical utility of implementing ML.

Methods: We recruited 5508 older ED patients ( $\geq 65$ years old) in three hospitals between 2009 and 2018. Patients were randomized into a 70\%/30\% split for model training and testing. Using 10 clinical variables from their electronic health records, a prediction model using the synthetic minority oversampling technique preprocessing algorithm was constructed to predict five outcomes.

Results: The best areas under the curves of predicting outcomes were: random forest model for hospitalization (0.840), pneumonia (0.765), and sepsis or septic shock (0.857), XGBoost for intensive care unit admission (0.902), and logistic regression for in-hospital mortality (0.889) in the testing data. The predictive model was further applied in the hospital information system to assist physicians' decisions in real time.
\end{abstract}

Conclusions: ML is a promising way to assist physicians in predicting outcomes in older ED patients with influenza in real time. Evaluations of the effectiveness and impact are needed in the future.

Keywords: Emergency department, Influenza, Hospital information system, Machine learning, Mortality, Older, Prediction, Random forest

\section{Background}

The rapidly aging population is one of the most important issues worldwide. In the United States, older adults ( $\geq 65$ years old) were $15.2 \%$ of the total population in 2016 , are projected to be $20 \%$ in 2030 , and $23.5 \%$ in

\footnotetext{
* Correspondence: chienchenghuang@yahoo.com.tw

${ }^{+}$Chung-Feng Liu and Chien-Cheng Huang contributed equally to this work. 'Department of Emergency Medicine, Chi Mei Medical Center, 901 Zhonghua Road, Yongkang District, Tainan City 710, Taiwan

${ }^{8}$ Department of Senior Services, Southern Taiwan University of Science and Technology, Tainan, Taiwan

Full list of author information is available at the end of the article
}

2060 [1]. Taiwan is one of the rapidly aging countries in the world. In 2018, the number of deaths was nearly equal to that of births [2]. Older adults represented 14\% of the total population in 2018, and are projected to be $20 \%$ in 2025 [2].

Influenza is a life-threatening disease for the older population. An Asian study revealed that older adults contributed to $70-90 \%$ of total deaths [3]. The mortality rate of influenza in older adults was nearly 39 -fold that of the population aged 40-64 years old [3]. Influenzarelated complications, including cardiorespiratory

C C The Author(s). 2021 Open Access This article is licensed under a Creative Commons Attribution 4.0 International License, which permits use, sharing, adaptation, distribution and reproduction in any medium or format, as long as you give appropriate credit to the original author(s) and the source, provide a link to the Creative Commons licence, and indicate if changes were made. The images or other third party material in this article are included in the article's Creative Commons licence, unless indicated otherwise in a credit line to the material. If material is not included in the article's Creative Commons licence and your intended use is not permitted by statutory regulation or exceeds the permitted use, you will need to obtain permission directly from the copyright holder. To view a copy of this licence, visit http://creativecommons.org/licenses/by/4.0/. The Creative Commons Public Domain Dedication waiver (http://creativecommons.org/publicdomain/zero/1.0/) applies to the data made available in this article, unless otherwise stated in a credit line to the data. 
diseases, pneumonia, chronic obstructive pulmonary disease (COPD), and ischemic heart diseases, are the common causes of death [3].

Because of limited medical resources during the influenza season, predicting outcomes in older adults with influenza and their subsequent disposition becomes a critical issue. In our previous study, we recruited 409 older patients with influenza for developing a Geriatric Influenza Death Score (GID score) [4]. This study identified five independent mortality predictors: severe coma (Glasgow Coma Scale [GCS] $\leq 8$ ), past histories of malignancy and coronary artery disease (CAD), elevated Creactive protein $(\mathrm{CRP})$ levels $(>10 \mathrm{mg} / \mathrm{dl})$, and bandemia (>10\% band cells) [4]. Three mortality risk and disposition groups were formed according to five predictors: (1) low risk (1.1\%; 95\% confidence interval [CI], 0.5-3.0\%); (2) moderate risk (16.7, 95\% CI, 9.3-28.0\%); and (3) high risk (40, 95\% CI, 19.8-64.2\%). The GID score has an area under the receiver operating characteristic curve of 0.86 , and Hosmer-Lemeshow goodness of fit of 0.578 [4].

Although the GID score is a potentially good clinical decision rule (CDR) in older adults with influenza, it has the limitations of the small size of derivation sample and lacks both automation and feedback in real time to clinicians [5]. Artificial intelligence (AI) is defined as that uses computer techniques, including machine learning $(\mathrm{ML})$ and deep learning (DL) to represent intelligent behavior [6]. In recent years, a great deal of evidence showed that AI could handle more variables that are already available through electronic health records (EMRs) and may better predict patient outcomes [5]. We performed searches on Google Scholar and PubMed using the keywords "AI," "death," "influenza," "machine learning," "mortality," "older adult," and "outcome," but we did not find any AI application in this field. Therefore, we conducted the present study for clarifying the issue and applying it in the hospital information system (HIS) to assist decision making in real time.

\section{Methods}

\section{Study design, setting, and participants}

We included emergency physicians, information engineers, data scientists, quality managers, and nurse practitioners to establish a multi-disciplinary team for this project (Fig. 1). After our literature review, we decided to use the previous study about predicting mortality in older ED patients with influenza as the main reference [4]. We identified all older patients ( $\geq 65$ years old) with influenza who visited the ED between January 1, 2009, and December 31, 2018, from the EMRs of three hospitals: Chi Mei Medical Center, Chi Mei Hospital, Liouying, and Chi Mei Hospital, Chiali. The present study hospitals are not the hospitals for developing the GID score. The criteria of influenza are defined as the diagnosis of International Classification of Diseases, Ninth Revision, Clinical Modification (ICD-9-CM) of 487 or 488 or a prescription of Oseltamivir, Peramivir, or Relenza in the index ED visit.

\section{Definitions of feature variables}

We adopted 10 potential risk factors proposed in the previous study for predicting mortality in the older patients with influenza as the feature variables for the ML or DL in this study [4]: (1) tachypnea (respiratory rate $>20 / \mathrm{min}$ ); (2) severe coma (GCS $\leq 8,3$ ) history of hypertension; (4) history of CAD; (5) history of malignancy; (6) bedridden; (7) leukocytosis (WBC > 12,000 cells/mm); (8) bandemia (>10\% band cells); (9) anemia (hemoglobin $<12 \mathrm{mg} / \mathrm{dL}$ ); and (10) elevated CRP (>10 mg/dL).

In addition, we also recruited age, sex, vital signs, and past histories of hypertension (ICD-9: 401-405), diabetes (ICD-9-CM: 250), COPD (ICD-9-CM: 496), CAD (ICD-9CM: 410-414), stroke (ICD-9: 436-438), malignancy (ICD-9: 140-208), congestive heart failure (CHF, ICD-9CM: 428), dementia (ICD-9: 290), bedridden, feeding with a nasogastric tube, and nursing home resident, laboratory data including white blood cell count (WBC), bandemia, hemoglobin, platelet, serum creatinine, CRP, procalcitonin, glucose, Na, K, GOT, and GPT for this study. The patients who did not have a record of subsequent follow-up were excluded. Missing laboratory data were treated as the normal values (i.e., respiratory rate: $12 / \mathrm{min}$, GCS: 15 , WBC: 7000 cells $/ \mathrm{mm}$, band form: $0 \%$, hemoglobin: $12 \mathrm{mg} /$ $\mathrm{dL}$, and CRP: $2.5 \mathrm{mg} / \mathrm{dL}$ ).

\section{Outcome measurements}

The outcome measurements were binary coded as the follows: (1) hospitalization; (2) complications with pneumonia (ICD-9-CM: 480-486): (3) complications with sepsis or septic shock (ICD-9-CM: 038, 790.7, 995.91, 995.91, 785.52); (4) admitted to intensive care unit (ICU); and (5) in-hospital mortality.

\section{Ethical statement}

The present study was approved and granted permission to access the raw data by the institutional review board in the Chi Mei Medical Center. Because this study is retrospective and it contains de-identified information, informed consent from the participants was waived. The waiver does not affect the rights and welfare of the participants.

\section{Data processing, model comparison, and application in the HIS}

First, we extracted, transformed, and validated the data from the HIS into a data mart. Missing and ambiguous data were carefully processed at this step. Second, we randomly split the data to two dataset $(70 \% / 30 \%)$ and used the synthetic minority oversampling technique (SMOTE) to 


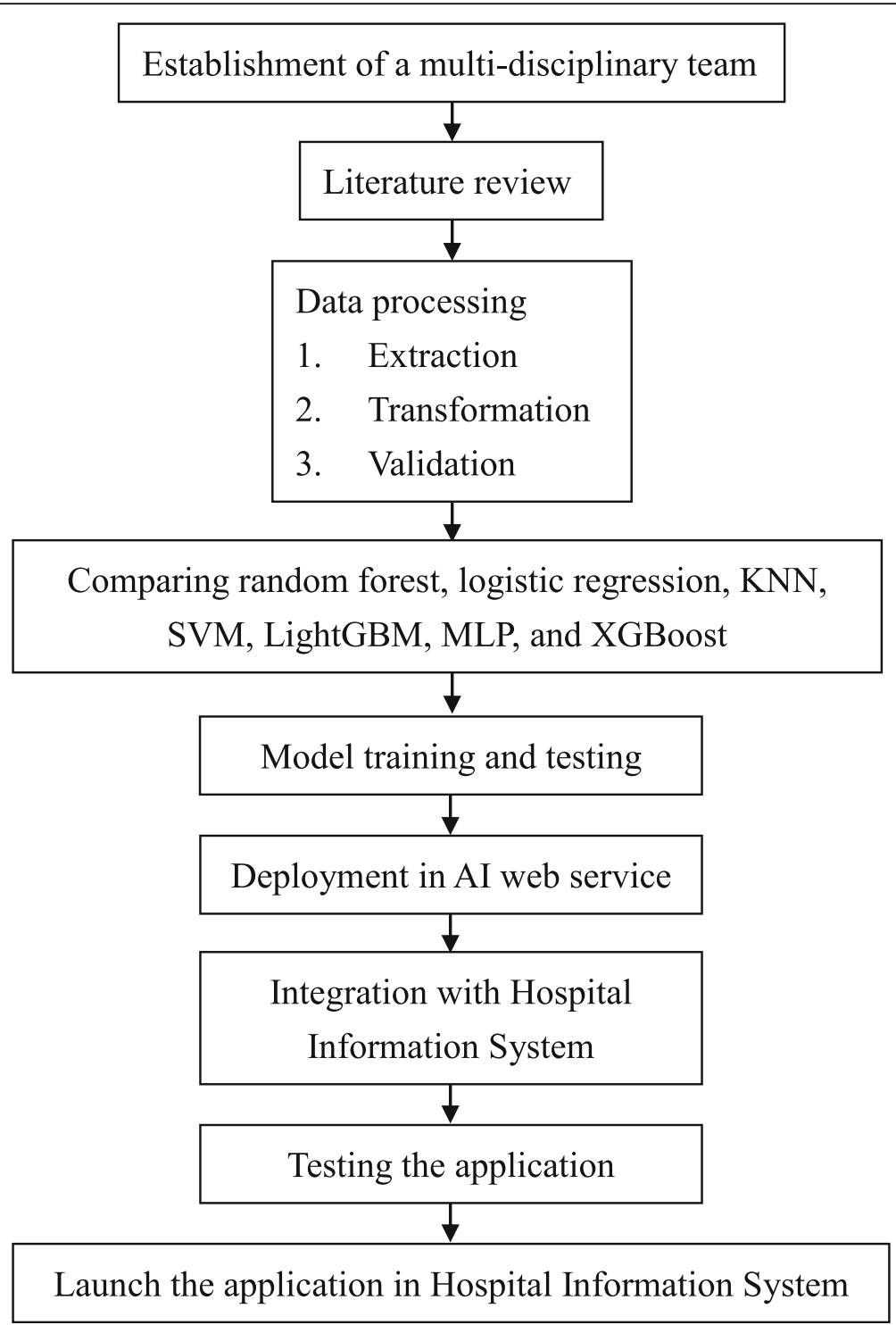

Fig. 1 Flowchart of the application of ML for predicting outcomes in older ED patients with influenza. ED, emergency department; KNN, Knearest neighbors; SVM, support vector machine; LightGBM, light gradient boosting machine; MLP, multilayer perceptron; XGBoost, Extreme Gradient Boosting; Al, artificial intelligence

enlarge the first dataset (70\%) as training dataset because of imbalanced outcome samples. The second dataset (30\%) is used as testing dataset without any resampling. Third, according the optimal modeling result with testing dataset, we compared accuracy, sensitivity, specificity, positive predictive value, negative predictive value, and the area under the curve (AUC) among the analyses of the random forest, logistic regression, K-nearest neighbors (KNN), support vector machine (SVM), light gradient boosting machine (LightGBM), multilayer perceptron (MLP, a kind of DL), and Extreme Gradient Boosting (XGBoost). In this step, we conducted grid search with hyper-parameters for each algorithm to obtain the optimal models (hyper-parameter ranges for each algorithm were summarized in Supplementary Table 1). Then, we selected the best algorithm to develop the prediction model for each outcome. Fourth, we deployed the model in the AI web service and integrated it with the HIS in the ED. After twomonths of pilot testing and validating, we launched the prediction application in the HIS to assist physicians for decision making in real time.

\section{Patient and public involvement}

Patients and the public were not be involved in this study. 


\section{Results}

In total, we recruited 5508 older ED patients into the present study. The mean \pm standard deviation (SD) of age was $76.61 \pm 7.44$ years old, and the female proportion was $50.67 \%$ (Table 1). The proportion of the three age subgroups were young elderly (43.06\%), moderately elderly $(40.56 \%)$, and old elderly (16.38\%). The mean \pm SD of respiratory rate and GCS were $19.16 \pm 3.94$ breaths/ min and $14.41 \pm 1.84$, respectively. The histories of ED patients were hypertension (56.05\%), CAD (19.64\%), malignancy (14.32\%), and bedridden (31.94\%). The mean \pm SD of WBC, hemoglobin, and CRP were $8670.00 \pm 4220.00$ cells $/ \mathrm{mm}^{3}, 12.42 \pm 1.95 \mathrm{mg} / \mathrm{dL}$, and $42.06 \pm 50.98 \mathrm{mg} / \mathrm{dL}$, respectively. The proportions of patient outcomes were hospitalization (47.33\%), complications with pneumonia (37.71\%), complications with sepsis or septic shock (5.57\%), admitted to ICU (1.07\%), and in-hospital mortality (2.20\%).

Comparisons of predictive accuracies among the random forest, logistic regression, KNN, SVM, LightGBM, MLP, and XGBoost revealed that the random forest model had the best AUC for hospitalization, pneumonia, and sepsis or septic shock than did other models in the testing dataset (Table 2 and Supplementary Fig. 1). The XGBoost had the best AUC for ICU admission (0.902) and logistic regression had the best AUC for in-hospital mortality (0.889). Table 3 summarized the best AUC for each outcome in the testing dataset, which was adopted for building the prediction model in further. Feature importance according to a random forest, logistic regression, LightGBM, and XGBoost for predicting the five outcomes was also reported (Supplementary Fig. 2).

We applied the best algorithm for predicting outcomes in older ED patients in the HIS to assist decision making in real time. An AI button was set up in the HIS of the ED (Supplementary Fig. 3). When the clinician presses the AI button, the AI application automatically catch the feature variables from the HIS and pops up a screen of the prediction result within $1 \mathrm{sec}$ (Supplementary Fig. 4). The prediction result shows a personalized prediction for hospitalization, complications with pneumonia, complications with sepsis or septic shock, admitted to ICU, and in-hospital mortality. Using five-level Likert, a mean of 4.6 was responded by 101 times of use, which indicates that the AI prediction is useful for the clinicians.

\section{Discussion}

The present study revealed that the random forest had the best AUC for predicting hospitalization, pneumonia, and sepsis or septic shock, XGBoost had the best AUC for predicting ICU admission, and logistic regression had the best AUC for predicting in-hospital mortality in older ED patients with influenza. The predictions are very fast, in real time, and actionable, which provide
Table 1 Characteristics of older ED patients with influenza in this study

\begin{tabular}{|c|c|}
\hline Variable & Total patients $(n=5508)$ \\
\hline Age (years) & $76.61 \pm 7.44$ \\
\hline \multicolumn{2}{|l|}{ Age subgroup (\%) } \\
\hline Young elderly (65-74) & 43.06 \\
\hline Moderately elderly (75-84) & 40.56 \\
\hline Old elderly ( $\geq 85$ ) & 16.38 \\
\hline \multicolumn{2}{|l|}{ Sex, \% } \\
\hline Female & 50.67 \\
\hline Male & 49.33 \\
\hline \multicolumn{2}{|l|}{ Triage vital signs } \\
\hline GCS & $14.41 \pm 1.84$ \\
\hline $\mathrm{SBP}(\mathrm{mm} \mathrm{Hg})$ & $142.88 \pm 32.77$ \\
\hline Heart rate (beats/min) & $93.38 \pm 24.24$ \\
\hline Respiratory rate (breaths/min) & $19.16 \pm 3.94$ \\
\hline Body temperature $\left({ }^{\circ} \mathrm{C}\right)$ & $37.53 \pm 6.64$ \\
\hline \multicolumn{2}{|l|}{ Past histories (\%) } \\
\hline Hypertension & 56.05 \\
\hline Diabetes & 32.37 \\
\hline COPD & 12.87 \\
\hline CAD & 19.64 \\
\hline CVA & 18.77 \\
\hline Malignancy & 14.32 \\
\hline $\mathrm{CHF}$ & 11.27 \\
\hline Dementia & 10.62 \\
\hline Bedridden & 31.94 \\
\hline \multicolumn{2}{|l|}{ Laboratory data } \\
\hline WBC (cells/mm³) & $8670.00 \pm 4220.00$ \\
\hline Bandemia (\%) & $4.10 \pm 5.24$ \\
\hline Hemoglobin (mg/dL) & $12.42 \pm 1.95$ \\
\hline Platelet $\left(10^{3} / \mathrm{mm}^{3}\right)$ & $187.36 \pm 72.39$ \\
\hline Creatinine (mg/dL) & $1.29 \pm 1.52$ \\
\hline hs-CRP (mg/dL) & $42.06 \pm 50.98$ \\
\hline Sodium (mEq/L) & $134.68 \pm 4.86$ \\
\hline Potassium (mmol/L) & $3.76 \pm 0.52$ \\
\hline GOT (U/L) & $51.55 \pm 172.64$ \\
\hline GPT (U/L) & $31.79 \pm 64.43$ \\
\hline \multicolumn{2}{|l|}{ Outcomes (\%) } \\
\hline Hospitalization & 47.33 \\
\hline Pneumonia & 37.71 \\
\hline Sepsis or septic shock & 5.57 \\
\hline ICU admission & 1.07 \\
\hline In-hospital mortality & 2.20 \\
\hline
\end{tabular}

Data are presented as mean \pm SD or percent. ED Emergency department; GCS Glasgow coma scale; SBP Systolic blood pressure; COPD Chronic obstructive pulmonary disease; $C A D$ Coronary artery disease; CVA Cerebrovascular accident; CHF Congestive heart failure; WBC White blood cell count; $h s-C R P$ High sensitivity C-reactive protein; GOT Glutamic oxaloacetic transaminase; GPT Glutamate pyruvate transaminase; ICU Intensive care unit; SD Standard deviation 
Table 2 Comparisons of predictive accuracies among random forest, logistic regression, KNN, SVM, LightGBM, MLP, and XGBoost in the outcomes of testing dataset of older ED patients with influenza

\begin{tabular}{|c|c|c|c|c|c|c|}
\hline Outcomes and predictive models & Accuracy & Sensitivity & Specificity & PPV & NPV & AUC \\
\hline \multicolumn{7}{|l|}{ Hospitalization } \\
\hline Random forest & 0.769 & 0.744 & 0.791 & 0.762 & 0.775 & 0.840 \\
\hline Logistic regression & 0.737 & 0.751 & 0.726 & 0.711 & 0.764 & 0.799 \\
\hline KNN & 0.736 & 0.737 & 0.736 & 0.715 & 0.757 & 0.790 \\
\hline SVM & 0.750 & 0.751 & 0.749 & 0.728 & 0.770 & 0.840 \\
\hline LightGBM & 0.748 & 0.714 & 0.780 & 0.744 & 0.752 & 0.823 \\
\hline MLP & 0.733 & 0.702 & 0.760 & 0.724 & 0.740 & 0.806 \\
\hline XGBoost & 0.721 & 0.705 & 0.736 & 0.706 & 0.735 & 0.800 \\
\hline \multicolumn{7}{|l|}{ Pneumonia } \\
\hline Random forest & 0.679 & 0.681 & 0.679 & 0.562 & 0.778 & 0.765 \\
\hline Logistic regression & 0.662 & 0.661 & 0.662 & 0.542 & 0.764 & 0.709 \\
\hline KNN & 0.645 & 0.700 & 0.613 & 0.522 & 0.771 & 0.683 \\
\hline SVM & 0.657 & 0.700 & 0.631 & 0.534 & 0.777 & 0.733 \\
\hline LightGBM & 0.653 & 0.700 & 0.625 & 0.530 & 0.775 & 0.724 \\
\hline MLP & 0.660 & 0.660 & 0.660 & 0.540 & 0.762 & 0.688 \\
\hline XGBoost & 0.674 & 0.700 & 0.658 & 0.553 & 0.784 & 0.744 \\
\hline \multicolumn{7}{|l|}{ Sepsis or septic shock } \\
\hline Random forest & 0.795 & 0.750 & 0.798 & 0.179 & 0.982 & 0.857 \\
\hline Logistic regression & 0.799 & 0.750 & 0.801 & 0.182 & 0.982 & 0.832 \\
\hline KNN & 0.714 & 0.750 & 0.712 & 0.133 & 0.980 & 0.785 \\
\hline SVM & 0.707 & 0.750 & 0.705 & 0.130 & 0.980 & 0.806 \\
\hline LightGBM & 0.739 & 0.739 & 0.739 & 0.143 & 0.980 & 0.822 \\
\hline MLP & 0.730 & 0.728 & 0.730 & 0.137 & 0.979 & 0.761 \\
\hline XGBoost & 0.744 & 0.739 & 0.744 & 0.146 & 0.980 & 0.811 \\
\hline \multicolumn{7}{|l|}{ ICU admission } \\
\hline Random forest & 0.860 & 0.722 & 0.862 & 0.054 & 0.996 & 0.885 \\
\hline Logistic regression & 0.720 & 0.778 & 0.719 & 0.030 & 0.997 & 0.867 \\
\hline KNN & 0.607 & 0.611 & 0.607 & 0.017 & 0.993 & 0.622 \\
\hline SVM & 0.768 & 0.778 & 0.768 & 0.036 & 0.997 & 0.778 \\
\hline LightGBM & 0.809 & 0.722 & 0.810 & 0.040 & 0.996 & 0.874 \\
\hline MLP & 0.629 & 0.611 & 0.629 & 0.018 & 0.993 & 0.649 \\
\hline XGBoost & 0.912 & 0.722 & 0.914 & 0.085 & 0.997 & 0.902 \\
\hline \multicolumn{7}{|l|}{ In-hospital mortality } \\
\hline Random forest & 0.792 & 0.806 & 0.792 & 0.079 & 0.995 & 0.875 \\
\hline Logistic regression & 0.816 & 0.806 & 0.816 & 0.089 & 0.995 & 0.889 \\
\hline KNN & 0.652 & 0.639 & 0.652 & 0.039 & 0.988 & 0.663 \\
\hline SVM & 0.789 & 0.722 & 0.791 & 0.071 & 0.992 & 0.762 \\
\hline LightGBM & 0.769 & 0.722 & 0.770 & 0.065 & 0.992 & 0.844 \\
\hline MLP & 0.675 & 0.667 & 0.675 & 0.044 & 0.989 & 0.728 \\
\hline XGBoost & 0.751 & 0.806 & 0.750 & 0.067 & 0.994 & 0.858 \\
\hline
\end{tabular}

KNN K-nearest neighbors; SVM Support vector machine; LightGBM Light gradient boosting machine; MLP Multilayer perceptron, XGBoost Extreme Gradient Boosting; ED Emergency department; PPV Positive predictive value; NPV Negative predictive value; AUC Area under the curve 
Table 3 Evaluation report using the best model with the SMOTE preprocessing algorithm on the outcomes of testing dataset of older ED patients with influenza

\begin{tabular}{|c|c|c|c|c|c|c|c|c|c|}
\hline Outcome & Number & $\begin{array}{l}\text { Negative } \\
\text { outcome }\end{array}$ & $\begin{array}{l}\text { Positive } \\
\text { outcome }\end{array}$ & Accuracy & Sensitivity & Specificity & PPV & NPV & AUC \\
\hline Hospitalization (random forest) & 5508 & 2901 & 2607 & 0.769 & 0.744 & 0.791 & 0.762 & 0.775 & 0.840 \\
\hline Pneumonia (random forest) & 5508 & 3431 & 2077 & 0.679 & 0.681 & 0.679 & 0.562 & 0.778 & 0.765 \\
\hline Sepsis or septic shock (random forest) & 5508 & 5201 & 307 & 0.795 & 0.750 & 0.798 & 0.179 & 0.982 & 0.857 \\
\hline ICU admission (XGBoost) & 5508 & 5449 & 59 & 0.912 & 0.722 & 0.914 & 0.085 & 0.997 & 0.902 \\
\hline In-hospital mortality (logistic regression) & 5508 & 5387 & 121 & 0.816 & 0.806 & 0.816 & 0.089 & 0.995 & 0.889 \\
\hline
\end{tabular}

SMOTE Synthetic minority oversampling technique; ED Emergency department; PPV Positive predictive value; NPV Negative predictive value; AUC Area under the curve; ICU Intensive care unit; XGBoost Extreme Gradient Boosting

prognostic information to assist in decision making, including disposition and outcome explanation.

Using AI prediction for assisting decision-making is an appealing idea [7]. Because of the increased availability of EMRs and advancement of computer performance and algorithm, AI prediction based on the medical big data becomes a promising way for healthcare [8]. In recent years, the rapid progression of cloud and IoT (internet of things) by healthcare monitor and wearable sensor networks also greatly support the development of real-time AI prediction [8]. Therefore, the AI-based tools, which are designed to improve diagnosis, care planning, and outcome will be incorporated into healthcare services in the near future [9]. Many regulations about AI use in healthcare need to be developed, including establishment of normative standard, evaluation guidelines, and monitoring and reporting systems [9].

The adopted feature variables in this study, including comorbidities and abnormal vital signs and laboratory data, are the risk factors for poor outcomes. The more feature variables, the poorer outcome in the result of AI prediction.

The random forest is superior to the traditional model (i.e., logistic regression) for developing CDR in predicting hospitalization, pneumonia, sepsis or septic shock, and ICU admission. One possible reason for the lower predictive accuracies of logistic regression is that it lacks external validation [5]. Traditional CDRs, including the GID score, are typically developed by gathering data at one or more hospitals, and then using both to derive and validate a model from a chosen set of predictors. The developed CDRs are then used in other hospitals, different from the original study hospital [10]. A recent study reviewed 127 new prediction models and showed that external independent validation was uncommon in predictive models [11]. Predictive performance in external validation tends to be worse than the original study [11]. In contrast to the GID score derived from other hospitals, we used local real-world big data in multicenters to make predictions about the local population, which improves accuracy over the traditionally derived model. The variables used in the present study are structured data from the local EMR without being subjected to ambiguous clinical definitions or biases of data collection.

The random forest model is an ensemble learning method for classification and regression [12, 13]. It combines many binary decision trees, which are built by several bootstrapped learning samples, and chooses a subset of variables randomly at each node $[12,13]$. Each tree in the random forest will vote for some input $\mathrm{x}$, then the voting majority of trees will determine the output of the classifier [14]. The random forest can use a large number of trees in the ensemble to handle high dimensional data [14]. The random forest is a common method adopted for predicting outcomes and selecting predictors in the ED. A study about predicting in-hospital mortality in ED patients with sepsis revealed that the AUC of the random forest was 0.86, superior to the CART (classification and regression tree) model (0.69); logistic regression model (0.76); CURB-65 (Confusion, Urea, Respiratory rate, Blood pressure plus age $\geq 65$ years old) (0.73); MEDS (mortality in emergency department sepsis) (0.71); and mREMS (modified rapid emergency medicine score) (0.72) [5]. A study used the random forest to select the most relevant variables for major adverse cardiac events in ED patients with chest pain [12]. They found that the selection predictor by the random forest is promising in discovering a few relevant and significant predictors [12].

The SMOTE adopted in the present study is the most common and effective method of oversampling for adjusting imbalanced data [15]. SMOTE solves the problems of both high-class skew and high sparsity and works in the "feature space" rather than "data space" [16]. By taking each minority class sample and the K-nearest neighbors, SMOTE creates synthetic samples for effectively forcing the decision region of the minority class to become more general [16]. Without duplicating the data, SMOTE increases the data space and amplifies the features of the minority class [16]. Studies with SMOTE preprocessing in health care are also acceptable $[17,18]$.

According to our literature review, the present study has the strength of being the first real-time prediction 
application in the HIS using ML for older ED patients with influenza. The limitations are as follows. First, interpretability and inferences about variables are the problems of ML. Second, we did not compare the predictive accuracy between this model and the physician's judgment. Further studies about this issue, as well as the impact of this model, are warranted. Third, variable selection was not conducted in this study. We decided to adopt 10 potential risk factors proposed in the previous study for increasing the explainability for AI models. In the future, including as many variables as possible and reducing the number by running proper variable selection algorithms are needed. Fourth, the application may not be generalized to other hospitals because it needs building an infrastructure to make real-time predictive analytics a reality.

\section{Conclusions}

We developed the first real-time prediction application in the HIS for predicting outcomes in older ED patients with influenza using a big data-driven and machine learning approach. This real-time prediction is a promising way to assist the physician's decision making and explanations to patients and their families. Further studies about the predictive accuracy between this model and both the physician's judgment, impact of the application, and including as many variables as possible and reducing the number by running proper variable selection algorithms are needed.

\section{Abbreviations \\ ED: Emergency department; ML: Machine learning; DL: Deep learning; COPD: Chronic obstructive pulmonary disease; GID: Geriatric influenza death; GCS: Glasgow coma scale; CAD: Coronary artery disease; CRP: C-Reactive protein; Cl: Confidence interval; CDR: Clinical decision rule; Al: Artificial intelligence; EMRs: Electronic medical records; HIS: Hospital information system; ICD-9-CM: International classification of diseases, ninth revision, clinical modification; ICU: Intensive care unit; SMOTE: Synthetic minority oversampling technique; AUC: Area under the curve; KNN: K-nearest neighbors; SVM: Support vector machine; LightGBM: Light gradient boosting machine; MLP: Multilayer perceptron; XGBoost: Extreme gradient boosting; SD: Standard deviation; CART: Classification and regression tree; CURB- 65: Confusion, urea, respiratory rate, blood pressure plus age $\geq 65$ MEDS: Mortality in emergency department sepsis; mREMS: Modified rapid emergency medicine score}

\section{Supplementary Information}

The online version contains supplementary material available at https://doi. org/10.1186/s12877-021-02229-3.

\section{Additional file 1.}

\section{Acknowledgments}

We thank Miss Yu-Shan Ma for statistical assistance and Enago for the English revision.

\section{Authors' contributions}

THT, CCH (2nd author), CFL, and CCH (corresponding author) designed and conceived this study and wrote the manuscript. CJC and TLL performed the data processing, deployment in Al web service, integration with HIS, testing the application, and launching the application in the HIS. CFL performed model training and testing and statistical analysis. SLH, HJL, and JJW provided professional discussions and suggestions and wrote the manuscript. All authors read and approved the final manuscript.

\section{Funding}

This work was supported by Chi Mei Medical Center, [Grant number CMFH R108119]. The funding body played no role in the design of the study and collection, analysis, and interpretation of data and in writing the manuscript.

\section{Availability of data and materials}

The datasets used during the current study are available from the corresponding author on reasonable request.

\section{Declarations}

Ethics approval and consent to participate

The present study was approved and granted permission to access the raw data by the institutional review board in the Chi Mei Medical Center. Because this study is retrospective and it contains de-identified information, informed consent from the participants was waived. The waiver does not affect the rights and welfare of the participants.

Consent for publication

Not applicable.

\section{Competing interests}

All authors denied any financial and non-financial competing interests.

\section{Author details}

'Department of Emergency Medicine, Chi Mei Medical Center, 901 Zhonghua Road, Yongkang District, Tainan City 710, Taiwan. ${ }^{2}$ Department of Biotechnology, Southern Taiwan University of Science and Technology, Tainan, Taiwan. ${ }^{3}$ Information Systems, Chi Mei Medical Center, Tainan, Taiwan. ${ }^{4}$ Department of Nursing, Chi Mei Medical Center, Tainan, Taiwan. ${ }^{5}$ Department of Emergency Medicine, Taipei Medical University, Taipei, Taiwan. ${ }^{6}$ Department of Medical Research, Chi Mei Medical Center, Tainan, Taiwan. ${ }^{7}$ Allied Al Biomed Center, Southern Taiwan University of Science and Technology, Tainan, Taiwan. ${ }^{8}$ Department of Senior Services, Southern Taiwan University of Science and Technology, Tainan, Taiwan. ${ }^{9}$ Department of Environmental and Occupational Health, College of Medicine, National Cheng Kung University, Tainan, Taiwan.

Received: 5 October 2020 Accepted: 19 April 2021

Published online: 27 April 2021

\section{References}

1. An Aging Nation: Projected Number of Children and Older Adults. https:// www.census.gov/library/visualizations/2018/comm/historic-first.html.

2. Population Projections for R.O.C. Taiwan: 2016;2060. https://pop-proj.ndc. gov.tw/main_en/dataSearch.aspx?uid=78\&pid=78.

3. Wong CM, Chan KP, Hedley AJ, Peiris JS. Influenza-associated mortality in Hong Kong. Clin Infect Dis. 2004;39(11):1611-7. https://doi.org/10.1 086/425315.

4. Chung JY, Hsu CC, Chen JH, Chen WL, Lin HJ, Guo HR, et al. Geriatric influenza death (GID) score: a new tool for predicting mortality in older people with influenza in the emergency department. Sci Rep. 2018;8(1): 9312. https://doi.org/10.1038/s41598-018-27694-6.

5. Taylor RA, Pare JR, Venkatesh AK, Mowafi H, Melnick ER, Fleischman W, et al. Prediction of in-hospital mortality in emergency department patients with Sepsis: a local big data-driven, machine learning approach. Acad Emerg Med. 2016;23(3):269-78. https://doi.org/10.1111/acem.12876.

6. Battineni G, Sagaro GG, Chinatalapudi N, Amenta F. Applications of machine learning predictive models in the chronic disease diagnosis. J Pers Med. 2020;10(2):21.

7. Chumbita M, Cilloniz C, Puerta-Alcalde P, Moreno-Garcia E, Sanjuan G, Garcia-Pouton N, et al. Can artificial intelligence improve the management of pneumonia. J Clin Med. 2020;9(1):248.

8. Alpert JS. The electronic medical record: beauty and the beast. Am J Med. 2019;132(4):393-4. https://doi.org/10.1016/j.amjmed.2018.12.004. 
9. Esmaeilzadeh P. Use of Al-based tools for healthcare purposes: a survey study from consumers' perspectives. BMC Med Inform Decis Mak. 2020; 20(1):170. https://doi.org/10.1186/s12911-020-01191-1.

10. Toll DB, Janssen KJ, Vergouwe $Y$, Moons KG. Validation, updating and impact of clinical prediction rules: a review. J Clin Epidemiol. 2008;61(11): 1085-94. https://doi.org/10.1016/j.jclinepi.2008.04.008.

11. Siontis GC, Tzoulaki I, Castaldi PJ, loannidis JP. External validation of new risk prediction models is infrequent and reveals worse prognostic discrimination. J Clin Epidemiol. 2015;68(1):25-34. https://doi.org/10.1016/j. jclinepi.2014.09.007.

12. Liu N, Koh ZX, Goh J, Lin Z, Haaland B, Ting BP, et al. Prediction of adverse cardiac events in emergency department patients with chest pain using machine learning for variable selection. BMC Med Inform Decis Mak. 2014; 14(1):75. https://doi.org/10.1186/1472-6947-14-75.

13. Genuer R, Poggi JM, Tuleau-Malot C. Variable selection using random forests. Pattern Recogn Lett. 2010;31(14):2225-36. https://doi.org/10.1016/j. patrec.2010.03.014

14. Khalilia M, Chakraborty S, Popescu M. Predicting disease risks from highly imbalanced data using random forest. BMC Med Inform Decis Mak. 2011; 11(1):51. https://doi.org/10.1186/1472-6947-11-51.

15. Wang Y, Wu X, Chen Z, Ren F, Feng L, Du Q. Optimizing the predictive ability of machine learning methods for landslide susceptibility mapping using SMOTE for Lishui City in Zhejiang Province, China. Int J Environ Res Public Health. 2019;16(3):368

16. Taft LM, Evans RS, Shyu CR, Egger MJ, Chawla N, Mitchell JA, et al. Countering imbalanced datasets to improve adverse drug event predictive models in labor and delivery. J Biomed Inform. 2009;42(2):356-64. https:// doi.org/10.1016/j.jbi.2008.09.001.

17. Sui Y, Wei Y, Zhao D. Computer-aided lung nodule recognition by SVM classifier based on combination of random undersampling and SMOTE. Comput Math Methods Med. 2015;2015:368674.

18. Kuo CY, Yu LC, Chen HC, Chan CL. Comparison of models for the prediction of medical costs of spinal fusion in Taiwan diagnosis-related groups by machine learning algorithms. Healthc Inform Res. 2018;24(1):29-37. https:/ doi.org/10.4258/hir.2018.24.1.29.

\section{Publisher's Note}

Springer Nature remains neutral with regard to jurisdictional claims in published maps and institutional affiliations.

Ready to submit your research? Choose BMC and benefit from:

- fast, convenient online submission

- thorough peer review by experienced researchers in your field

- rapid publication on acceptance

- support for research data, including large and complex data types

- gold Open Access which fosters wider collaboration and increased citations

- maximum visibility for your research: over $100 \mathrm{M}$ website views per year

At $\mathrm{BMC}$, research is always in progress.

Learn more biomedcentral.com/submissions 